Revue

Revue de l'histoire des religions

de Ihistoire

des religions

$3 \mid 2011$

Varia

Pour une archéologie du rite. Nouvelles perspectives de l'archéologie funéraire, études réunies par John SCHEID

Rome, École française de Rome, 2008, 358 p.

Hélène Bernier-Farella

\title{
OpenEdition
}

Journals

Édition électronique

URL : http://journals.openedition.org/rhr/7798

DOI : $10.4000 /$ rhr.7798

ISSN : 2105-2573

Éditeur

Armand Colin

Édition imprimée

Date de publication : 1 septembre 2011

Pagination : 439-442

ISBN : 978-2200-92727-9

ISSN : 0035-1423

Référence électronique

Hélène Bernier-Farella, «Pour une archéologie du rite. Nouvelles perspectives de l'archéologie funéraire, études réunies par John scheID », Revue de l'histoire des religions [En ligne], 3 | 2011, mis en ligne le 09 décembre 2011, consulté le 22 septembre 2020. URL : http://journals.openedition.org/rhr/7798; DOI : https://doi.org/10.4000/rhr.7798

Ce document a été généré automatiquement le 22 septembre 2020.

Tous droits réservés 


\section{Pour une archéologie du rite. Nouvelles perspectives de l'archéologie funéraire, études réunies par John SCHEID}

Rome, École française de Rome, 2008, 358 p.

Hélène Bernier-Farella

\section{RÉFÉRENCE}

Pour une archéologie du rite. Nouvelles perspectives de l'archéologie funéraire, études réunies par John scheid, Rome, École française de Rome, 2008, 358 p., $29 \mathrm{~cm}$ («Collection de l'École française de Rome » 40), $88 €$

1 L'ouvrage se présente comme la première moisson d'études issues du programme européen Vivre et mourir dans l'Empire romain (coordonné par la Surintendance archéologique de Rome, et animé notamment par l'EFR, le Collège de France et l'Université de Cambridge : le lien indiqué du site web du projet est rompu, mais celui de Porta Nocera sera visité avec profit: < http://www.mourirapompei.net/index.html $>$ ). Il confirme l'importance de ce projet pour l'archéologie funéraire romaine et pour l'archéologie rituelle en général.

2 Les vingt-trois études richement illustrées concernent aussi bien l'Italie, la Gaule Belgique et la Britannia, avec une attention particulière pour deux fouilles exemplaires, celles de l'enclos de Vesonius de Porta Nocera à Pompéi (S. Lepetz, W. Van Andringa) et celles de Classe, près de Ravenne (M.-G. Maioli, J. Ortali, J.Scheid), selon une problématique unique qui vise à lire la tombe, au-delà de sa monumentalité et des interactions sociales qui s'y déroulent, comme un lieu de culte rituel. Un paradigme unitaire renforce la cohérence méthodologique de l'ensemble, celui de la recherche de l'exhaustivité absolue des données et de la réalité du rituel. J. Ortali expose ainsi que la nécropole doit être fouillée comme un tout organique, même dans ses semblances de vides. La «monostrate» apparente des fosses doit laisser place à une restitution de 
chaque sol, aussi labiles soient-ils, au moyen d'une microstratigraphie dont la méthode de fouille est clairement exposée et dont l'intérêt est également explicité par M. Witteyer pour les tombes de la région de Mainz. L'étude globale de l'espace de la nécropole est également préconisée par C. Gaeng et J. Metzler qui montrent comment l'attention portée aux abords de la sépulture permet parfois de recomposer le rituel autrement ignoré.

3 Afin de retrouver chaque geste du rituel et de traiter l'ensemble du matériel, une collaboration organique entre archéologues, archéozoologues, anthropologues, céramologues, carpologues et épigraphistes sur le lieu même de la fouille est préconisée, comme c'est le cas à Porta Nocera ou à Classe. À défaut, le bénéfice et le modus operandi d'associations interdisciplinaires moins larges sont largement illustrés et forment le plus gros de l'ouvrage, surtout dans l'association entre archéologie et examen du matériel osseux. Des progrès notables sont ainsi acquis dans l'interprétation de structures funéraires jusqu'alors ambiguës, comme les fosses à cendres (aschengruben) étudiées par A. Abegg Wigg ou les bûchers à fosses (V. Bel, F. Blaizot, H. Duday). La collaboration entre archéologues et anthropologues associés dans l'étude de trois mausolées de la nécropole romaine de Collatina (A. Buccelato et alii) permet de supposer l'existence de rites funéraires hors du commun, comme la réunion dans un même enclos sur deux siècles d'individus montrant des malformations physiques. L'attention pour les restes osseux et ce qu'ils peuvent livrer d'informations rituelles passe aussi par l'étude taphonomique des tombes, (étude de la transformation des corps après leur enfouissement), qui permet de reconstruire la présence de cercueils, l'exposition éventuelle des corps avant la mise au tombeau ou de retrouver les aménagements internes des tombes, mêmes les plus ténus, comme un coussin dans une tombe d'enfant à Pompéi (H. Duday). Les éléments ligneux, donc périssables, retrouvés par cette méthode sont confirmés par les fouilles de Classe, dont les conditions exceptionnelles - une couche scellée par une strate d'argile et un milieu anaérobique ont permis la conservation de cercueils, planches et coussins funèbres en bois (C. Leoni, M.G. Maioli, G. Montevecchi). Le même type d'examen sur le matériel animal permet de recomposer de façon précise, comme le montre P. Méniel, les parts des viandes découpées offertes au mort, dans une visée sacrificielle et celles calcinées sur le bûcher ou celles déposées telles quelles dans les tombes, selon des processus que les textes ne renseignent pas en détail.

4 L'exhaustivité recherchée des traces rituelles aboutit en second lieu à une attention très poussée pour les méthodes de prélèvement du matériel. La plupart des interventions insistent sur ce point, recommandant jusqu'au prélèvement intégral du sédiment de petites unités de fouille ou des fosses funéraires dans leur ensemble (V Bel, F. Blaizot, H. Duday) afin de déterminer les éventuelles manipulations dont le corps fut l'objet, les modalités de dépôts des offrandes et leur emplacement exact, précisions rituelles autrement indisponibles pour les incinérations. V. Zech-Matterne recommande ainsi pour les restes carpologiques un prélèvement à la fois manuel, à vue, et celui d'unités entières de sédiments, ces deux méthodes s'enrichissant et se compensant mutuellement. Les modalités du prélèvement, du stockage et de la restauration des objets funéraires sont également exposées en détail par M. Angelini et alii qui montrent l'intérêt de l'utilisation, ici encore, de la fouille très fine et font bénéficier les archéologues confrontés à une fouille funéraire de nombreux protocoles techniques visant à restituer les détails les plus fugaces. La tentative de restitution globalisante de la fouille se lit aussi à travers la recherche de moyens exhaustifs 
d'enregistrement, pendant logique du prélèvement systématique de l'ensemble du matériel.

5 Les méthodes les plus systématiques de relevés et l'homogénéisation de l'enregistrement du matériel à des fins de restitution globale et de comparaison sont enfin exposées dans trois études. Ces relevés se matérialisent, comme D. Joly le montre pour l'enregistrement informatique de la fouille de Porta Nocera et G. Montevecchi pour celle de Classe, par des systèmes informatiques généralisables à d'autres sites et qui permettent d'archiver sur une base de données unique l'ensemble des informations de fouilles (matériel, descriptions mais aussi relevés graphiques et photographies). P. Booth et A. Boyle montrent comment le cadre d'une archéologie privatisée et son contrôle par la législation britannique ont permis une homogénéisation et un niveau majeur de précision des relevés des fouilles funéraires, permettant, pour le site de Lankhills, l'enregistrement digital des objets et le remplacement des croquis par l'usage de la photographie géo-rectifiée.

6 Les méthodes préconisées et explicitées dans l'ouvrage permettent un réel progrès dans la connaissance des actes rituels et le sous-titre "nouvelles perspectives» de l'archéologie funéraire est ainsi pleinement justifié. Ces méthodes semblent cependant difficiles à généraliser et $\mathrm{P}$. Méniel le remarque à juste titre, en raison de leur coût financier élevé et de la lenteur des processus engagés. Les avancées dont témoigne l'ouvrage montrent que cette documentation totalisante n'est pas une illusion, surtout pour les sites où toutes les conditions peuvent être réunies (Pompéi et Classe). L'on peut toutefois se demander si la volonté de récolter et d'analyser l'ensemble des données repérables par chaque spécialiste ne conduit pas à une fragmentation de la réalité historique du rituel et si cette recherche de l'exhaustivité, malgré la richesse des résultats obtenus, ne conduit pas à en paralyser l'effort d'interprétation. L'étude de J.Pearce est représentative de ce biais, lorsque, en soulignant les distorsions inhérentes à la généralisation de résultats issus d'échantillons archéologiques, nécessairement partiels, il met en garde contre toute généralisation (p. 39). Il est cependant manifeste que pour donner un sens aux phénomènes étudiés, la généralisation, malgré la perte d'exactitude qu'elle engendre, est un processus indispensable.

7 Aucune étude du recueil n'a ainsi pour objet la question du renouvel-lement du sens du rituel. À titre d'exemple, alors qu'à Classe, l'on apprend que l'espèce dominante déposée sur les tombes est le pin, qu'à Pompéi il s'agit du figuier et de la vigne, la valeur symbolique de ces espèces n'est pas questionnée. Ce qui ne choque en rien dans une étude carpologique, très riche au demeurant, manque cependant à l'édifice dans son ensemble. On peut donc regretter que les gains majeurs obtenus dans la connaissance des rites ne se soient pas enrichis d'une relecture de ses dimensions symboliques et religieuses, alors même que les eschatologies antiques ont récemment fait l'objet d'études renouvelées. La lecture globale du rituel exposée par S. Martin-Kilcher détaille les gestes et l'enjeu social des funérailles mais non leur arrière-plan religieux. Or le culte des défunts, que le rituel met en place - ce qui est bien souligné par W. Van Andringa et S. Lepezt - peut difficilement se comprendre hors des représentations religieuses qui le sous-tendent et qu'il contribue lui-même à définir. Si les textes anciens montrent effectivement leurs limites pour éclairer le rituel (J. Scheid, Prologue) il n'est pas certain que la recomposition précise des séquences rituelles puisse se suffire à elle-même dans la quête du sens des funérailles romaines. L'ajout de l'anthropologie 
sociale au bel ensemble de spécialités convoquées ici pourrait sans doute en enrichir la compréhension.

AUTEUR

HÉLÈNE BERNIER-FARELLA

Université de Cergy-Pontoise 